\title{
A dynamic optimization model for school network planning
}

\author{
António Antunes ${ }^{\mathrm{a}, *}$, Dominique Peeters ${ }^{\mathrm{b}}$ \\ ${ }^{a}$ Departamento de Engenharia Civil, Universidade de Coimbra, Coimbra, Portugal \\ ${ }^{\mathrm{b}}$ CORE \& Unité de Géographie, Université Catholique de Louvain, 1348 Louvain-la-Neuve, Belgium
}

\begin{abstract}
This paper describes a dynamic (multi-period) optimization model that has recently been used in Portugal to formulate planning proposals for the evolution of several school networks. This effort follows the extension of elementary education from 6 to 9 years as decided by the Government in 1986, and gradually implemented since then. The model allows for facility closure or size reduction, as well as facility opening and size expansion, with sizes limited to a set of pre-defined standards. It assumes facility costs are divided into a fixed component and two variable components, dependent, respectively, on capacity and attendance. (C) 2000 Elsevier Science Ltd. All rights reserved.
\end{abstract}

Keywords: Facility location; School networks; Capacity expansion

\section{Introduction}

One of the functions of any government, at the central or local level, is to provide the community within its jurisdiction with certain goods and services that would not otherwise be available (or not to the extent that they should) without its intervention. Education is one of those services, and surely one of foremost importance - if importance can be evaluated by the amount of collective concern and public spending assigned to the educational sector. Schools are the physical infrastructures used to produce educational services. In conjunction with

\footnotetext{
* Corresponding author. Tel.: + 351-39-857068; fax: + 351-39-822511.
}

E-mail address: antunes@dec.uc.pt (A. Antunes).

0038-0121/00/\$ - see front matter (C) 2000 Elsevier Science Ltd. All rights reserved.

PII: S0038-0121(99)00014-2 
teachers, who supply labor for the process, they constitute the crucial production factors used in this sector.

The central subject of this paper is school network planning, whereby "school network" we mean the set of schools through which education is provided to a given community. In the second section, the main elements of the corresponding problems are identified and discussed. The third section includes a survey of the models developed with regard to those problems, while the fourth proposes a new dynamic (multi-period) optimization model. The model allows for facility closure or size reduction, as well as facility opening and size expansion. The fifth section contains information about the path followed by the authors to solve the model, which eventually led to using simulated annealing. The sixth section presents the results obtained with the model for some Portuguese school networks, where expectations concerning demand evolution suggested a dynamic approach. In the seventh section, the policy implications of the model are analyzed. The paper ends with a synthesis of the main conclusions reached with this study and a presentation of research directions to be followed in the future.

\section{Problem statement}

School network planning problems consist of determining the school network needed to serve a given territory in terms of the educational demand in that same territory over the short, medium and long runs.

The solution to this kind of problem gives information about where and when new schools should be built, what their sizes should be, which schools should be kept open and which ones should be closed. Additionally, for the schools remaining open, it identifies which schools should maintain the same size and which should be resized, becoming larger or smaller.

The main decisions to be made regard the short term. In fact, what one really wants to know is what to do next. Decisions regarding the medium or long term are less important, because they may generally be changed. In a continuous planning framework, they will at least be reevaluated, and possibly modified. Short-term decisions are thus critical due to the twoway connection linking what will (or should) happen now to what will (or should) happen later. They must, therefore, be logical in light of future demands.

The ultimate goal of public facility planning is to satisfy demand (as much as possible). When it is possible to satisfy all potential users, the objectives will generally involve maximizing (socio-economic) benefits or, which is basically the same, minimizing costs. Others include maximizing accessibility (for given infrastructure costs) and maximizing equity. These objectives may, of course, conflict to various extents.

Achievement of the objectives may be subject to several constraints, e.g., capacity limits (upper and lower), budget provision, maximum catchment (i.e., the distance between users and the facility to which they are assigned should not exceed some given value), design standards, etc.

In most practical applications (including the ones presented in this paper), demand is assumed to be concentrated in a given number of "centers" - points representing the municipalities or towns of a region, or the neighborhoods of a town. Supply is assumed 
possible at a given number of "sites" — points representing either specific plots of land or approximate locations within a region, town or neighborhood.

The evolution of demand is often taken to be known, and to be independent of supply. Deterministic approaches take just one reference or project value for demand in each center and period. This value is relatively easy to calculate for education, particularly at the elementary level where attendance is compulsory. Stochastic approaches work with alternative scenarios wherein each one is associated with an estimated probability.

\section{Literature review}

Planning problems like those stated above, with explicit consideration of time issues, have been discussed by several authors, originating from two somewhat distinct operations research fields: "dynamic facility location" and "multi-regional capacity expansion". Research on the first began with Roodman and Schwartz [1,2]. One of the most representative models in this field was developed by Van Roy and Erlenkotter [3]. Erlenkotter [4,5] is credited, along with Manne, for having started research on the latter. They were followed by Fong and Srinivasan [6], Jacobsen [7], and Shulman [8], among others.

The Van Roy and Erlenkotter formulation is a mixed-integer linear programming model with an objective-function that minimizes total (discounted) socio-economic costs. Setup, operation and transport costs are considered. The objective-function adds fixed costs to variable costs dependent on the number of users. The model includes constraints to guarantee that:

- Demand from any center will be met, in each period, by some facility (or facilities).

- Demand will be satisfied at open sites only (i.e., sites where facilities are located).

- Sites will change their state, from open to closed or closed to open, at most once (a proposal indicating, for example, that a facility should be abandoned soon after being installed would hardly deserve much credibility).

Constraints aimed at ensuring that maximum or minimum capacity limits will be satisfied can be added to this list, though it should be noted here that, in the presence of this type of constraint, the method proposed to solve the model would no longer be the most appropriate (see Shulman [8]).

The model proposed in [3] is undoubtedly useful in dealing with practical school network planning problems, but like other "dynamic facility location" models, it has a major drawback: costs are implicitly assumed to depend on facility attendance, and not on capacity. However, at the least, setup costs will depend exclusively on the maximum number of users the facility will be able to accommodate, and not, of course, on the actual number it will have to accommodate in each period.

This drawback is absent from the "multi-regional capacity expansion" models, and particularly in the Fong and Srinivasan [6] model mentioned above. Its objective-function, also expressing cost minimization, distinguishes between costs dependent on attendance and costs dependent on capacity.

Nonetheless, this type of model allows neither open facilities to be closed, nor their capacity, calculated for each period by adding previous capacity expansions to the initial capacity, to be 
decreased. And, this may be a serious weakness in the presence of decreasing demand conditions, such as those encountered by elementary education today, at least in the Western World.

The literature on facility planning contains several articles dealing specifically with school networks, including those by Beguin et al. [9], Greenleaf and Harrison [10], Henig and Gerschak [11], Pizzolato [12], Tewari and Jena [13], and Viegas [14]. The models presented by Greenleaf and Harrison and by Viegas derive from a multi-period perspective, and are therefore closely related to the problem stated at the outset.

The Greenleaf and Harrison approach, motivated by a 32 center $\times 11$ site problem defined for the "State College Area School District", Pennsylvania (USA), uses a model which has some points in common with the one presented below. The approach is extremely detailed in handling financing aspects. But it is excessively simplistic in the way it treats school capacity, as it assumes that schools either remain open at the initial capacity or close.

The Viegas approach, motivated by a 37 center $\times 45$ site problem defined for the Municipality of Amadora, in the suburbs of Lisbon, Portugal, involves a combination of two models. The first seeks the minimization of construction costs subject to a maximum walking distance and capacity constraints. The second model seeks the minimization of walking distance costs (average or some weighted sum) subject to a maximum construction cost and capacity constraints. The approach may be quite useful in dealing with problems in areas presenting high population growth rates (like those observed in Amadora, during the 1970s and the 1980s), but it does not allow closures or size reductions of schools.

\section{A new model}

Due to the limitations previously noted, the existing models were not fully appropriate to formulate planning proposals for school networks in Portugal following the extension of elementary education from 6 to 9 years, as decided by the Government in 1986.

This led the authors to develop a new dynamic model having, among other features, the following:

- Over the planning horizon, each site will not change its state (open to closed or closed to open) more than once, and the corresponding facility may go through either size expansions or size reductions, but not both.

- Both setup and operation costs have a component dependent on capacity and a component dependent on attendance.

- Facility size is limited to pre-defined standards, expressed in terms of an architectural module, for instance, a given number of classrooms. (Note that modularity is also consistent with teaching staff management rules, defined as pupil/teacher ratios, followed in many countries.)

The corresponding formulation is as follows:

$$
\min C=\sum_{j \in \mathbf{J}} \sum_{k \in \mathbf{K}} \sum_{m \in \mathbf{M}} c_{v x_{j k m}} x_{j k m}+\sum_{k \in \mathbf{K}} \sum_{m \in \mathbf{M}} c_{f_{k m}}\left(y_{k m}^{+}+y_{k m}^{-}\right)+\sum_{k \in \mathbf{K}} \sum_{m \in \mathbf{M}} c_{v z_{k m}}\left(z_{k m}^{+}-z_{k m}^{-}\right)
$$


subject to:

$$
\begin{aligned}
& \sum_{k \in \mathbf{K}} x_{j k m}=1, \forall j \in \mathbf{J}, m \in \mathbf{M} \\
& \sum_{j \in \mathbf{J}} u_{j m} x_{j k m} \leqslant q_{k 0}\left(y_{k m}^{+}+y_{k m}^{-}\right)+q\left(z_{k m}^{+}-z_{k m}^{-}\right), \forall k \in \mathbf{K}, m \in \mathbf{M} \\
& \left(q_{\min _{k}}-q_{k 0}\right) y_{k m}^{+} \leqslant q \cdot z_{k m}^{+} \leqslant\left(q_{\max _{k}}-q_{k 0}\right) y_{k m}^{+}, \forall k \in \mathbf{K}, m \in \mathbf{M} \\
& q \cdot z_{k m}^{-} \leqslant\left(q_{k 0}-q_{\min _{k}}\right) y_{k m}^{-}, \forall k \in \mathbf{K}^{\mathbf{1}}, m \in \mathbf{M} \\
& y_{k m}^{+}+y_{k m}^{-} \leqslant 1, \forall k \in \mathbf{K}, m \in \mathbf{M} \\
& y_{k, m-1}^{+} \leqslant y_{k m}^{+}, \forall k \in \mathbf{K}^{\mathbf{0}}, m \in \mathbf{M} \backslash\{1\} \\
& y_{k m}^{+} \leqslant y_{k, m-1}^{+}, \forall k \in \mathbf{K}^{\mathbf{1}}, m \in \mathbf{M} \backslash\{1\} \\
& y_{k m}^{-} \leqslant y_{k, m-1}^{-}, \forall k \in \mathbf{K}^{\mathbf{1}}, m \in \mathbf{M} \backslash\{1\} \\
& z_{k, m-1}^{+} \leqslant z_{k m}^{+}, \forall k \in \mathbf{K}, m \in \mathbf{M} \backslash\{1\} \\
& z_{k, m-1}^{-} \leqslant z_{k m}^{-}, \forall k \in \mathbf{K}^{\mathbf{1}}, m \in \mathbf{M} \backslash\{1\} \\
& x_{j k m} \geqslant 0, \forall j \in \mathbf{J}, k \in \mathbf{K}, m \in \mathbf{M} \\
& y_{k m}^{+}, y_{k m}^{-} \in\{0,1\}, k \in \mathbf{K}, m \in \mathbf{M} \\
& z_{k m}^{+}, z_{k m}^{-} \geqslant 0 \text { and integer, } \forall k \in \mathbf{K}, m \in \mathbf{M}
\end{aligned}
$$

where:

$\mathbf{J}$ : set of centers $(j=1, \ldots, J)$;

$\mathbf{K}$ : set of sites $(k=1, \ldots, K)$;

$\mathbf{K}^{0}$ : set of initially closed sites;

$\mathbf{K}^{1}$ : set of initially opened sites;

M: set of periods $(m=1, \ldots, M)$;

$x_{j k m}$ : fraction of users from center $j$ assigned to a facility located at site $k$ in period $m$; $y^{+} \mathrm{km}=1$ if an expanding facility is located at site $k$ in period $m ; y^{+} \mathrm{km}=0$ otherwise;

$y^{-} \mathrm{km}=1$ if a reducing facility is located at site $k$ in period $m ; y^{-} \mathrm{km}=0$ otherwise;

$z^{+} \mathrm{km}$ : accumulated capacity expansion of the facility located at site $k$ up to period $m$; 
$z^{-} \mathrm{km}$ : accumulated capacity reduction of the facility located at site $k$ up to period $m$;

$c_{v x_{j k m}}$ : discounted attendance-variable cost of a facility located at site $k$ in period $m$ per user of center $j$ (includes transport costs);

$c_{f_{k m}}$ : discounted fixed cost of a facility located at site $k$ in period $m$ (for initially opened facilities it includes the costs associated with a capacity of $q_{k 0}$ );

$c_{v z_{k m}}$ : discounted capacity-variable cost increase (or decrease) of expanding (or reducing) a facility located at site $k$ in period $m$;

$u$ : number of users located at center $j$ in period $m$;

$q_{k 0}$ : initial capacity of the facility located at site $k$;

$q$ : capacity of a module;

$q_{\max k}\left(\geqslant q_{k 0}\right)$ : maximum capacity of a facility located at site $k$.

$q_{\min k}\left(\leqslant q_{k 0}\right)$ : minimum capacity of a facility located at site $k$.

This is a complicated mixed-integer linear optimization model to which we gave a name well within the location analysis tradition: "Dynamic Modular Capacitated Facility Location Problem" (DMCFLP) model. It contains three types of decision variables: the " $x$ ", representing assignment decisions; the " $y$ ", representing location decisions; and the " $z$ ", representing capacity decisions.

Function (1) expresses the objective of minimizing the total discounted (socio-economic) costs of a set of facilities. Facility costs are divided into two parts: fixed costs; capacity-variable costs proportional to the number of modules; and attendance-variable costs proportional to the number of users, a significant part of which will normally consist of transport costs. Initial capacity-variable costs of existing facilities are taken as fixed costs, which explains the minus sign applied to variables $z^{-} \mathrm{km}$.

Constraints (2) ensure that, in each period, the demand of any center will be met by some facility or facilities. Constraints (3) guarantee that the capacity of each facility in each period, resulting from adding the initial capacity to the accumulated capacity expansion occurring up to the period, will be large enough to meet the demand assigned to it. Constraints (4a) and (4b) ensure that maximum and minimum capacity limits, defined to avoid or exploit technical and economic scale advantages or disadvantages, will be taken into account at both expanding and reducing facilities. Constraints (5) guarantee that capacities may be either expanded or reduced, but not both. Constraints (6) ensure that facilities opened at initially closed sites, once opened, will remain open. Constraints (7a) and (7b) guarantee that facilities closed at initially opened sites, once closed, will remain closed. Constraints (8) ensure that the capacity of expanding facilities will never decrease. Finally, constraints (9) guarantee that the capacity of reducing facilities will never increase.

\section{Model solving}

The model introduced in the previous section is a quite difficult one, something that we soon understood after attempting and failing to solve a few $10 \times 10 \times 3$ (centers $\times$ sites $\times$ periods) problems using branch-and-bound packages like SCICONIC, XPRESS-MP and CPLEX (early versions). Branch-and-bound (B-B) is the general method more frequently used to solve mixedinteger linear optimization models (see Nemhauser and Wolsey [15] and Winston [16]). 
These failed attempts led the authors to investigate the possibilities of specialized dual-based and Lagrangean relaxation exact methods. However, after spending some time on that task, it became quite clear that this kind of method would hardly be successful in the current case.

When exact methods fail, it is necessary to resort to heuristics. Adopting a heuristic method to solve an optimization model means that global optimal solutions will no longer be guaranteed. But, if the heuristic is properly designed, it can identify good, local optimum solutions.

Our first idea for a heuristic consisted of using what may be called a "myopic" approach to solve the model. With this approach, the first-period problem is solved without taking into account future-period demands. Then, the second-period problem is solved given the facility set identified for the first-period problem, and so forth.

This approach was tested by solving a representative set of DMCFLP models and comparing the corresponding results to those given by the "panoramic" approach implicit to our model (within which short-term planning decisions about capacities also reflect the longterm expected evolution for demand). The tests were conducted on $6 \times 6 \times 3$ problems, the largest we have been able to solve using B-B packages within a reasonable computing effort.

The results obtained through the "myopic" and "panoramic" approaches were quite close on many occasions, though not in the presence of severe capacity shortages and important demand decreases. Indeed, this was precisely the situation encountered in many Portuguese regions when the expansion of elementary education was decided (for details, see Antunes [17]).

At this point, we decided to try simulated annealing (SA), a method that appeared to be relatively simple to apply to our problem. Of course, we were conscious of the fact that tests on complex, large-constrained models had been somewhat disappointing at times, but there were not many promising options open to us. Simulated annealing is a random search heuristic that became known within the operations research community after being successfully applied to the traveling salesman problem and other classic, difficult optimization problems (see Aarts et al. [18] and Cerny [19]).

Like any other heuristic method, SA does not guarantee a global optimum solution. However, the strategy employed by the corresponding algorithms in searching the solution space, within which moves to worse solutions are not excluded under certain conditions (defined by what are called annealing parameters), often allows them to avoid getting trapped in local optima.

Prior to preparing a SA algorithm for the DMCFLP model, we applied SA in solving a representative sample of uncapacitated facility location problem (UFLP) models. We then compared the results with those obtained with ADD + INTERCHANGE. This is a fast, reliable local search heuristic first proposed by Kuehn and Hamburger [20] which only allows moves to better solutions. The uncapacitated facility location problem, also named simple plant location problem (SPLP) or uncapacitated warehouse facility location problem (UWLP), is one of the most studied discrete optimization problems (see Cornuéjols et al. [21] and Krarup and Pruzan [22]).

In synthesis, we found that, for small problems $(20$ centers $\times 20$ sites $)$, the SA algorithm produced worse results and took longer on average than did ADD + INTERCHANGE (even with a very careful choice of annealing parameters). But, large problems ( 80 centers $\times 80$ sites) were solved better and faster, indicating the ability of SA to deal with complexity (see Table 1). 
Table 1

Comparison between SA and ADD + INTERCHANGE results for UFLP problems

\begin{tabular}{lllll}
\hline $\begin{array}{l}\text { Problem Size } \\
\text { centers } \times \text { sites })\end{array}$ & $\begin{array}{l}\text { SA better than } \\
\text { ADD }+\end{array}$ & $\begin{array}{l}\text { ADD }+ \text { better } \\
\text { than SA }\end{array}$ & $\begin{array}{l}\text { ADD + equal } \\
\text { to SA }\end{array}$ & $\begin{array}{l}\text { CPU Time } \\
\text { SA/ADD }+\end{array}$ \\
\hline $20 \times 20$ & 5 & 106 & 14 & 1.87 \\
$40 \times 40$ & 14 & 84 & 27 & 1.06 \\
$80 \times 80$ & 50 & 38 & 37 & 0.64 \\
\hline
\end{tabular}

This finding was decisive in convincing the authors that SA could be a good option for solving the DMCFLP model.

Preparation of the SA algorithm for the DMCFLP model was completed in a rather short time, due to the experience acquired with the UFLP model. Full details regarding contents of the SA algorithm and its performance are contained elsewhere [17,23]. However, it should be mentioned here that tests comparing the results provided by SA with those obtained with B-B in solving a representative sample of $6 \times 6 \times 3$ problems were quite positive (the sample was the same as that used before to test the "myopic" approach).

As a matter of fact, average SA solutions taken for 5 random seeds (i.e., 5 different sets of random numbers) were inferior to the B-B solutions in 36 of 50 problems, but only in 17 was SA unable to find the optimum B-B solution. Moreover, in all these 17 problems, it came quite close to it, as the difference was always smaller than $1 \%$. In the remaining 33 problems, SA behaved at least as efficiently as B-B. The use of "at least" is justified because, surprisingly, in 9 occasions, our algorithm gave better solutions than did B-B. This is something that would be impossible if the corresponding optimum solutions were actually exact, as we expected them to be (see Table 2).

\section{Practical applications}

The model described above was recently used to formulate planning proposals for the evolution of several regional school networks in Central Portugal. One of these applications, developed for the secondary school ("escolas secundárias": ES) network of the Baixo Mondego region (see Fig. 1), is described in full below. Three other applications are also noted, but without entering into details, in order to illustrate how algorithm performance changes with problem size.

\subsection{The Baixo Mondego case study}

The Baixo Mondego region occupies an area of $5600 \mathrm{~km}^{2}$, distributed among 8 municipalities, and has a population of approximately 900,000 persons, about 100,000 of whom live in the university town of Coimbra (3rd largest Portuguese town, though far behind Lisbon and Oporto).

Data used to prepare the planning proposal for the Baixo Mondego ES network is presented 
Table 2

Comparison between SA and B-B results for DMCFLP problems

\begin{tabular}{lllrr}
\hline SA Run & $\begin{array}{l}\text { SA }>1 \% \text { better } \\
\text { than B-B }\end{array}$ & $\begin{array}{l}\text { SA } \leqslant 1 \% \text { better } \\
\text { than B-B }\end{array}$ & $\begin{array}{l}\text { SA equal } \\
\text { to B-B }\end{array}$ & $\begin{array}{l}\text { B-B } \leqslant 1 \% \text { better } \\
\text { than SA }\end{array}$ \\
\hline Worst & 1 & 5 & 6 & 21 \\
Average & 4 & 4 & 6 & 34 \\
Best & 4 & 5 & 24 & 17 \\
\hline
\end{tabular}


below. Standards are those defined by GEP Educação [24], the planning and research department of the Portuguese Ministry of Education.

(1) Centers

- Number: 8.

- Location: concentrated in principal towns.

- Demand: described in Table 3, where it can be seen that the number of students is expected to grow very quickly in the near future to attain a maximum in the year 2001, and to decline after that. Details on how the values displayed were calculated can be found in Antunes [17].

(2) Sites

- Number: 20, i.e., the number of existing schools (9) plus 11 new locations, 3 in Coimbra, 2 in Figueira da Foz and 1 in each one of the remaining 6 centers.

- Location: coinciding with centers.

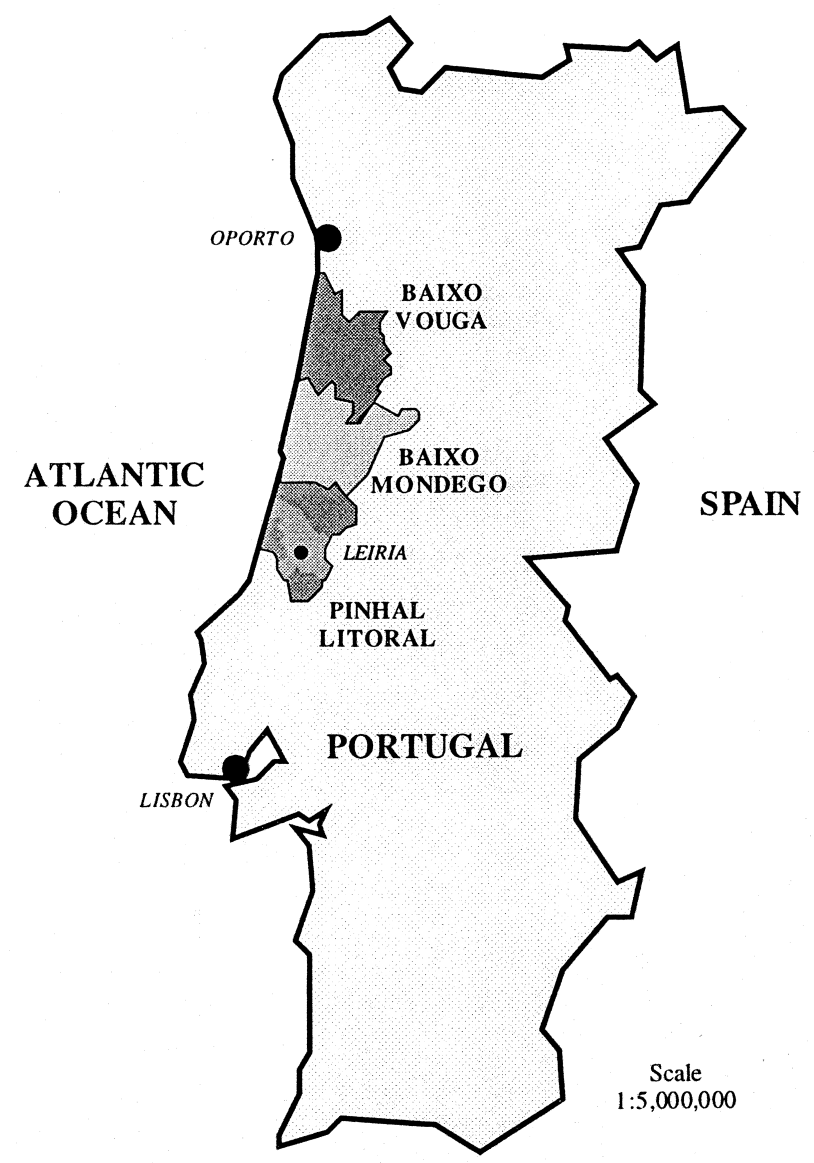

Fig. 1. The location of case study areas. 
(3) Capacities

(a) Classroom $=25$ students.

(b) Module, $q=6$ classrooms (150 students).

(c) Initially opened sites:

$-q_{k 0}$ : see Fig. 2, where it is shown that total initial capacity was equal to 9300 students in 1993, defining a short term deficit of 1125 students (45 classrooms).

$-q_{\min k}=\max \left\{q_{k 0}-2 q, \min \left(q_{k 0}, 3 q\right)\right\}, \forall k \in \mathbf{K}$.

$-q_{\max k}\left\{q_{k 0}, 8 q\right\}, \forall k \in \mathbf{K}$.

(d) Initially closed sites:

$-q_{\min k}=3 q, \forall k \in \mathbf{K}$.

$-q_{\max k}=6 q, \forall k \in \mathbf{K}$.

(4) Catchment: $d_{\max }=20 \mathrm{~km}$ (approximately 30 min using public transport).

(5) Costs (in $10^{6}$ Portuguese Escudos):

- Setup: fixed $=30$; variable $=60$ per module.

- Maintenance (over 5 years) $=20 \%$ of setup costs.

- Operation (over 5 years) $=90$ per classroom.

- Transport (over 5 years) $=0.080$ per user per $\mathrm{km}$.

- Discount rate (over 5 years) $=40 \%$.

The results obtained using DMCFLPAnnealing, a Think Pascal code developed for this study on a $40 \mathrm{MHz}$ Mac computer (Quadra 700), are summarized in Figs. 3-5, below, where two solutions for the Baixo Mondego problem are displayed. The first solution has been defined assuming the absence of budget constraints. The second solution assumes the presence of a short-term budget constraint amounting to $2 / 3$ of the setup expenditure identified for the first solution.

Table 3

Project values for ES students in Baixo Modego

\begin{tabular}{lrrr}
\hline Municipality & & ES students \\
& 1996 & 2001 & 2006 \\
\hline Cantanhede & 1164 & 1649 & 1438 \\
Coimbra & 4383 & 6252 & 5432 \\
Condeixa-a-Nova & 409 & 580 & 505 \\
Figueira da Foz & 1966 & 2822 & 2444 \\
Mira & 477 & 532 & 533 \\
Montemor-o-Velho & 823 & 1167 & 1017 \\
Penacova & 525 & 745 & 649 \\
Soure & 678 & 961 & 838 \\
Total & 10,425 & 14,710 & 12,856 \\
& & & \\
\hline
\end{tabular}


The solutions referred to above were the best obtained out of 5 SA runs, each one using a different set of random numbers.

Computer times for the 5 runs ranged between $45 \mathrm{~min}$ and $1 \mathrm{~h} 30 \mathrm{~min}$. Today, with a $200 \mathrm{MHz}$ machine, these values could easily be reduced by approximately $75 \%$.

\subsection{The "without budget constraint" solution}

The "without budget constraint" solution recommends short-term reduction of the capacity available at Coimbra and Figueira da Foz, and construction of four new schools, to be built at Mira, Montemor-o-Velho, Penacova and Soure. This means that the initial capacity deficit of 45 classrooms will be solved through a very significant decentralization of infrastructure, though Coimbra will continue to assist almost 500 outside students.

The new school in Mira will serve the students living in Mira as well as some from

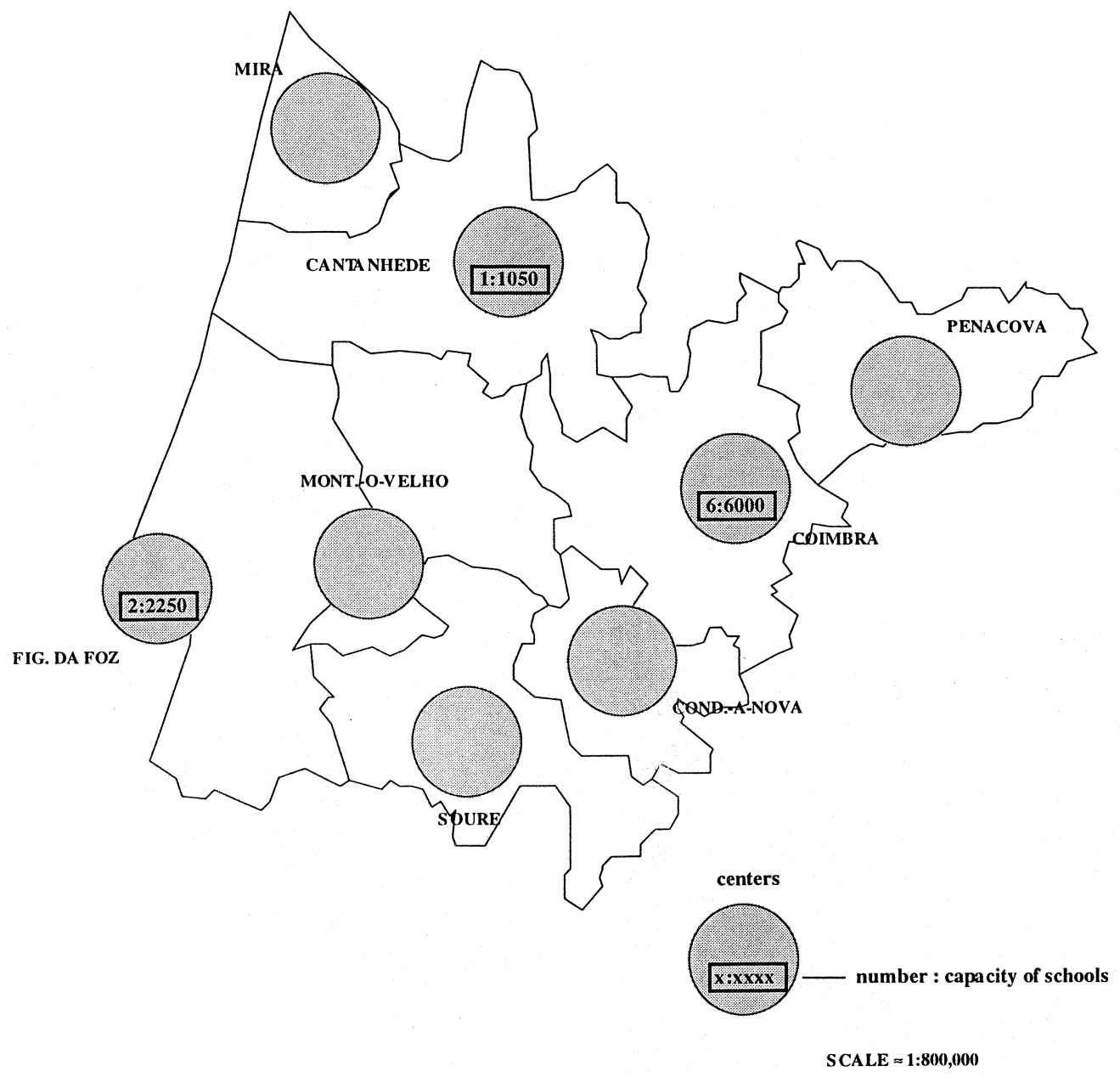

Fig. 2. The ES network of Baixo Mondego in 1993. 


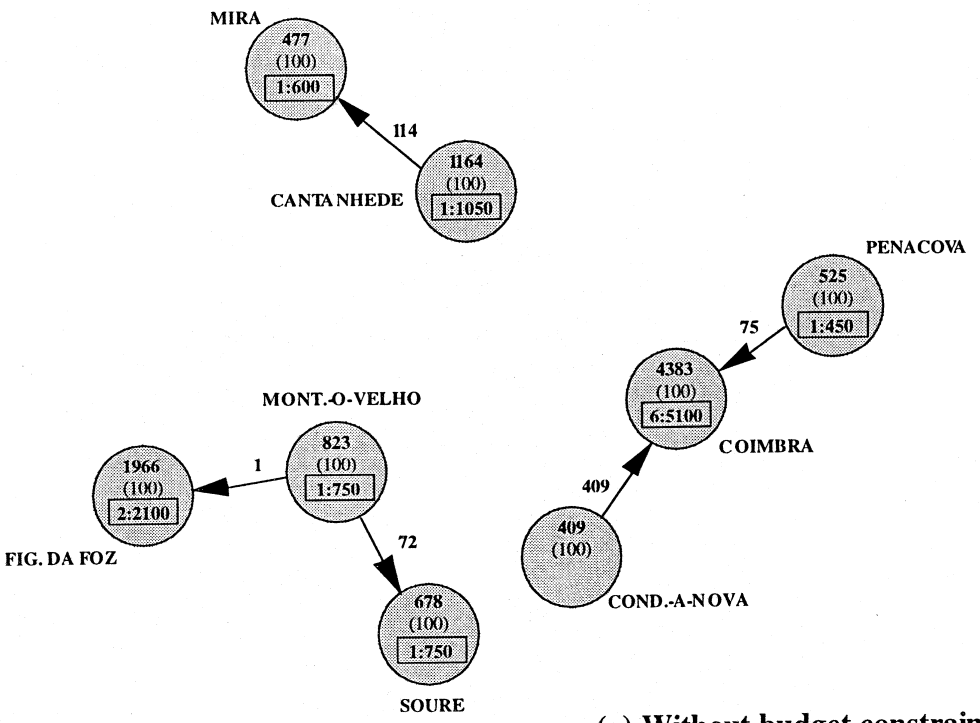

(a) Without budget constraint

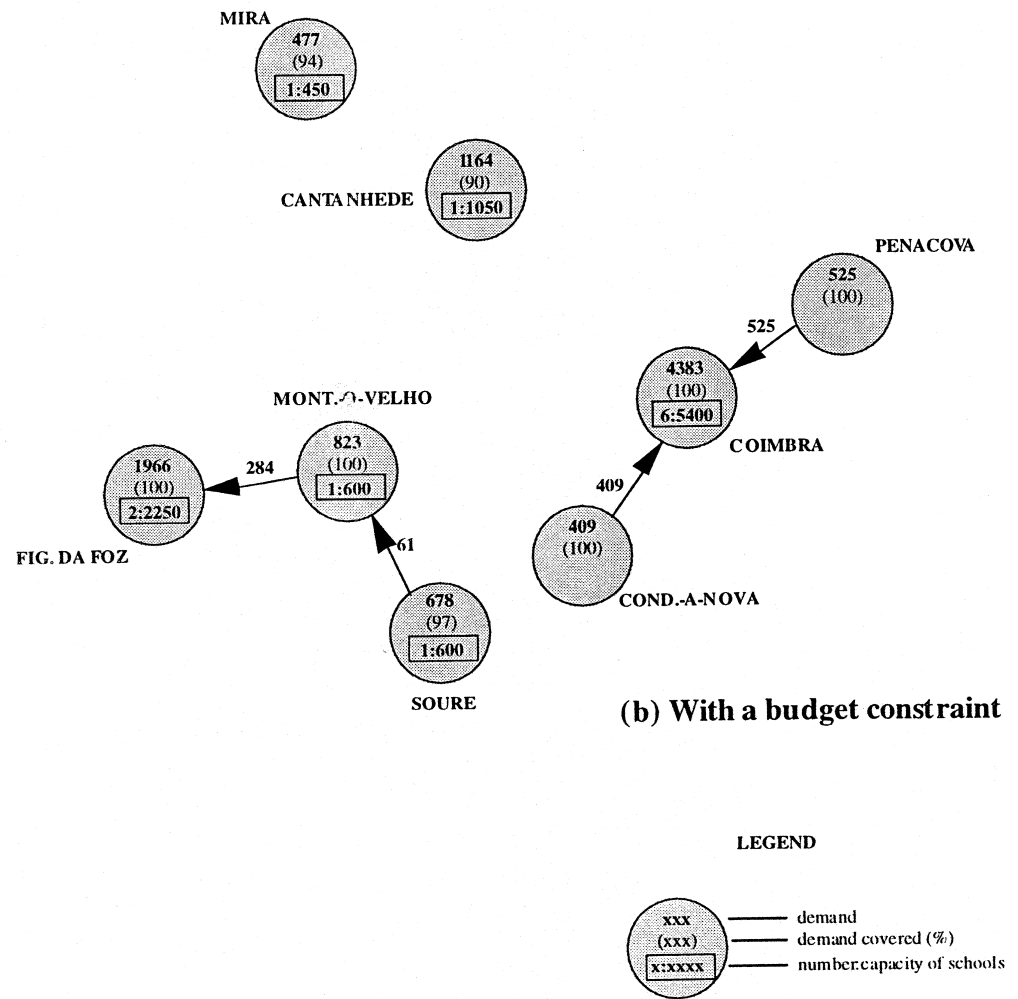

Fig. 3. Optimal short-term evolution for the Baixo Mondego ES network. 

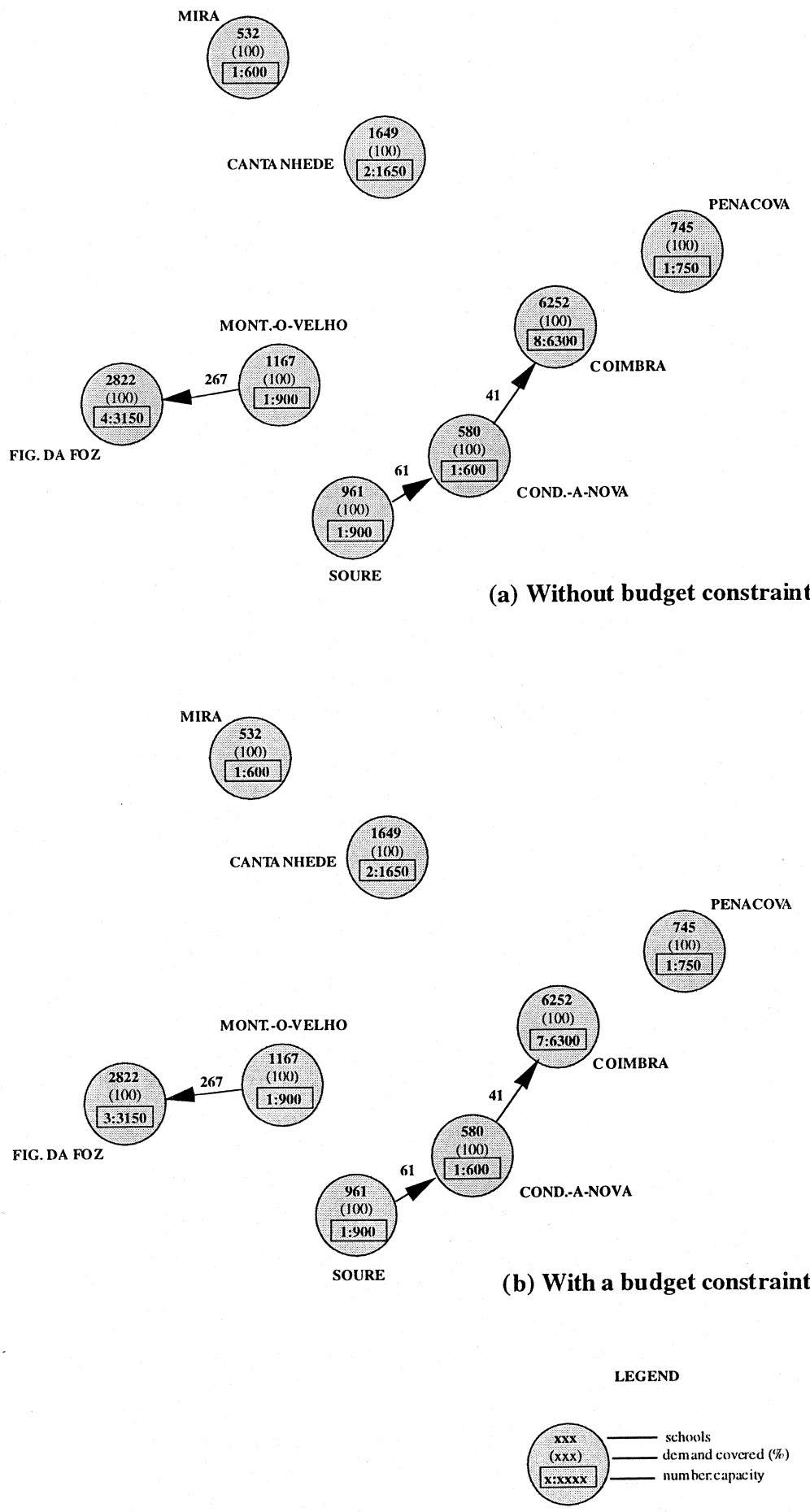

Fig. 4. Optimal medium-term evolution for the Baixo Mondego ES network. 


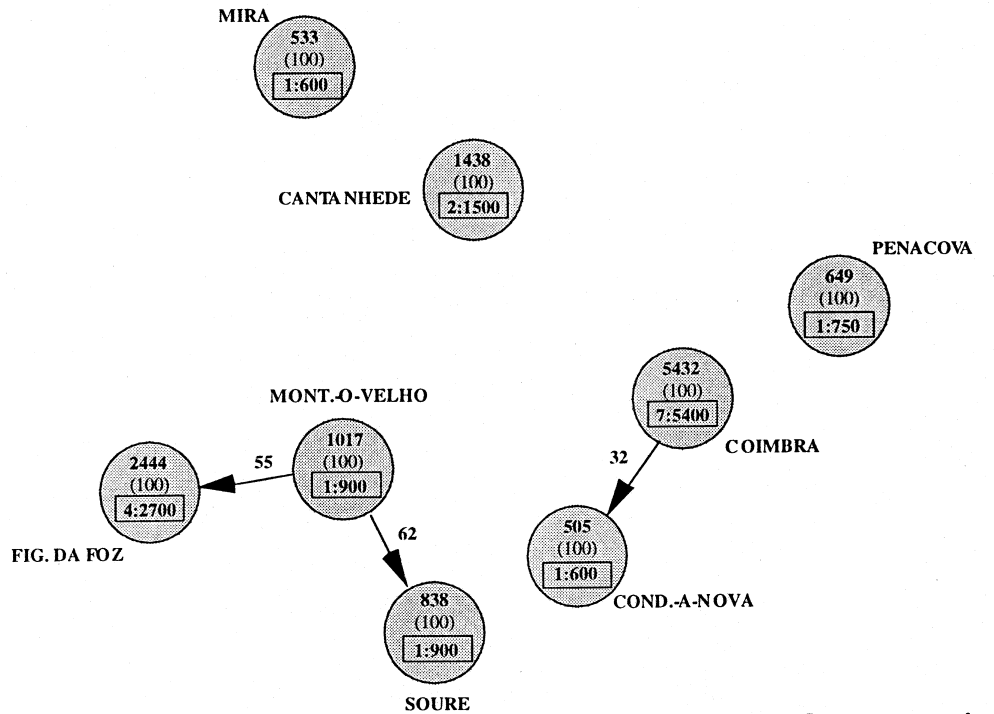

(a) Without budget constraint

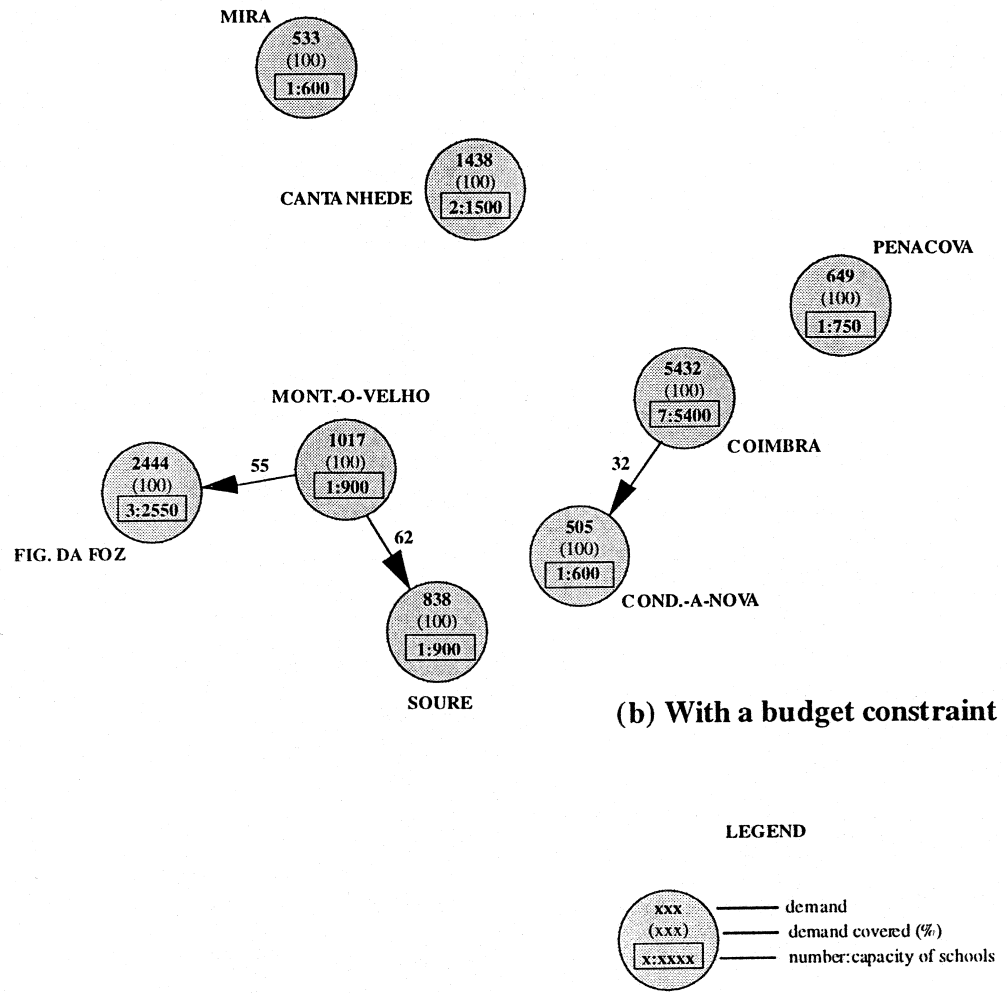

Fig. 5. Optimal long-term evolution for the Baixo Mondego ES network. 
Cantanhede (preferably, those living along the Cantanhede-Mira border which, as can be seen in Fig. 2, is quite close to Mira). The new school in Soure will also serve some Montemor-oVelho students since the new school in the latter municipality will not be large enough to satisfy the local demand.

It should be noted here that, in Portugal, the assignment of students to public schools is decided by the Administration, something that allows the assignment scheme to be easily implemented in practice.

In the medium-term, capacity will necessarily grow almost everywhere to meet the increase in demand induced by the extension of elementary education. This need will be met both by expanding some existing schools and, especially, by building six new schools. Of these, five will reinforce the infrastructure available in the main centers - Cantanhede, Coimbra and Figueira da Foz. The sixth will be located in Condeixa-a-Nova, the only municipality not served by a secondary school established in its own territory following the short-term intervention.

A peculiar aspect of the medium-term solution is the "chain effect" that links Soure, Condeixa-a-Nova and Coimbra. A problem may thus arise as many individuals will find it hard to understand why their children must attend a school outside their municipality in order to make room for students living in other municipalities. The reason behind this effect is, of course, the presence of accessibility constraints. If these constraints were absent, a better solution would likely accommodate all 580 Condeixa-a-Nova students in their own school while sending 20 Soure students to Condeixa and the remaining 41 to Coimbra. But, these students would have to travel too far according to the prevailing catchment rules (recall that the maximum catchment of a school is equal to $30 \mathrm{~min}$ using public transport).

In the long-term, capacity may be reduced because demand will fall as a consequence of the "fertility crisis" that has been strongly affecting Portugal these last years. This can be accomplished by decreasing supply at the main centers, by closing selected initially opened sites, and by reducing the capacity of others (recall that closure or size reduction of new schools was ruled out).

The proposed solution requires that some students travel to adjacent municipalities, but their numbers will decline with time. In the short-term, 671 students, i.e., $6.4 \%$ of the total, will have to so comply. Equivalent figures for the medium- and the long-term are, respectively, $2.5 \%$ and $1.2 \%$.

The evolution proposed for the Coimbra schools, summarized in Table 4, deserves special reference. In the short-term, as we mentioned before, there should be size reductions in some existing units in order to eliminate the excess supply created by construction of new schools in smaller communities. In the medium-term, however, the impact of expanding elementary education on secondary school demand will require two new schools. One of them would be required even without the previous size reductions. The other one would be unnecessary if these reductions were less significant. However, by solving the model it was found that building a new, smaller school would be better than expanding a big one. This happens because, according to prevailing decision rules, the big school would have to be kept afterwards, at its augmented capacity, under conditions of falling demand. The adjustment to long-term conditions is achieved by closing one of the initially existing schools, the smaller one, which was kept at initial capacity throughout the process, and reducing the capacity of two others as much as possible. 


\subsection{The "with a budget constraint" solution}

If we include a budget constraint, the solution, compared to the previous one, suggests: smaller short-term changes, with just three new smaller schools, at Mira, Montemor-o-Velho and Soure, and a smaller reduction at Coimbra (the situation regarding Figueira da Foz remains unchanged in this case). This means that there will still be some decentralization of infrastructure, but not as significant as before.

Available funds will be insufficient to satisfy all demand within the specified limits for school capacity and school catchment. $10 \%$ of Cantanhede users will thus need to be accommodated in classrooms with more than 25 students, or else attend a school more than $20 \mathrm{~km}$ away, for example, in Coimbra, where there will be some spare capacity. $6 \%$ of Mira users and 3\% of Soure users will be in similar conditions.

In the medium- and long-terms, the network will evolve in similar ways regardless of the presence or absence of a short-term budget constraint. The only differences will involve the infrastructure available at the main centers. The fact of not being able to reduce capacity initially (as budgetary factors limit the extension of short-term capacity transfers from large to small centers) makes it possible to concentrate the capacity required later on a smaller number of facilities. For example, in the medium-term, three instead of four schools will operate with a capacity of 3150 students at Figueira da Foz, while seven vs. eight schools will operate with a capacity of 6300 students at Coimbra.

\subsection{Other case studies}

Planning proposals were also formulated for the ES networks of Pinhal Litoral and Baixo Vouga and for the elementary school network of the largest municipality of the Pinhal Litoral region, Leiria (see Fig. 1).

Table 4

Proposed evolution for Coimbra schools

\begin{tabular}{lccrr}
\hline School & & & Capacity (users) \\
Initial & Short-term & & Long-term \\
\hline Existing & & & & 1050 \\
A & 1200 & 1050 & 900 & 900 \\
B & 1200 & 900 & 900 & 900 \\
C & 1200 & 900 & 900 & 750 \\
D & 1050 & 900 & 900 & 600 \\
E & 900 & 900 & 450 & $*$ \\
F & 450 & 450 & & 750 \\
New & $*$ & $*$ & 450 & 750 \\
I & $*$ & $*$ & 6300 & 450 \\
II & 6000 & 5100 & 5400 \\
Total & & & \\
\hline
\end{tabular}


The sizes of the models built for these scenarios were as follows (centers $\times$ sites): $5 \times 12$ (Pinhal Litoral); $12 \times 23$ (Baixo Vouga); and $29 \times 38$ (Leiria).

The CPU time required by Pinhal Litoral was quite small, never exceeding $20 \mathrm{~min}$ on the five annealing runs. For Baixo Vouga, it was clearly higher, attaining $4 \mathrm{~h}$ and $30 \mathrm{~min}$. But, this is insignificant when compared to the CPU time consumed for the Municipality of Leiria, which was well over $24 \mathrm{~h}$ for all five runs (on one occasion, almost 5 days).

\section{Policy implications}

The model presented in this paper contains several features that make it particularly wellsuited to deal with real-world public facility planning problems, particularly under complex demand conditions. The multi-period nature of the model is one of those features, possibly the most important one, as planning is more than simply deciding what a given system should be at a given point in time.

Application of the traditional, static approach suggests a future which is often (though not necessarily) expected to occur in the final planning period. The dynamic approach proposed in this paper, while keeping the medium- and long-terms in view, allows attention to be focused on the short-term. Instead of just an image of what the future should be at a particular moment, the dynamic approach also gives information on the appropriate trajectory to be followed.

The model may thus be an important tool in supporting the complex process of choosing the location and sizes of schools as well as other public facilities. It can also help with assignment decisions. However, the nearest-facility rule implicitly assumed by the model is not appropriate when deciding which students will attend a given school. Making assignments on a yearly basis according to user preferences is surely a better alternative (see Hanjoul and Peeters [25]).

By solving the model to optimality, it is possible to identify the best solution for the facility network under given accessibility, budget, capacity, and design specifications. Furthermore, it is possible to know the increase in costs associated with selecting a given solution (for the same specifications). At present, it is impossible to find guaranteed global optimum solutions for models involving anything but a very small number of centers and sites. In most real-world situations, it will thus be necessary to use heuristics, and be satisfied with a local optimum (which may be global or not).

At this point, it should be emphasized that solution of the proposed model does not translate to solution of the (real) problem. We think it should, instead, be seen as additional, important data to be used in the decision-making process, enabling all parties involved to have a deeper understanding of the implications of their options. For this reason, public participation will be much more critical to the planning process.

\section{Conclusion}

The main contribution of this paper involves specification of a dynamic optimization model for public facility planning. It was developed to respond to specific needs of school network 
planning in Portugal. The model allows for facility closures or size reductions, as well as facility openings and size expansions, with sizes limited to a set of pre-defined standards. It assumes facility costs to be divided into a fix component and two variable components, dependent, respectively, on capacity and attendance.

Solving this type of model remains a complex task. At present, the only feasible alternative involves heuristic methods. In this regard, simulated annealing has shown itself to be a useful approach. SA algorithms are quite easy to program and flexible to use, important features when it is necessary to adapt an existing algorithm to the peculiarities of a new, slightly different problem. Importantly, CPU time still is unreasonably high for problems involving over 30 centers, 30 sites and 3 periods. But, it must be said that we used a relatively simple algorithm. In the future, part of our research efforts will be focused on making the algorithm more sophisticated and efficient.

\section{Acknowledgements}

The authors acknowledge valuable critiques and suggestions received from Henri Zoller and Laurence Wolsey during the preparation of the Ph.D. dissertation upon which this paper is based, and from two anonymous referees. The first author also acknowledges the support received from Fundação Calouste Gulbenkian and Universidade de Coimbra while he was in Louvain.

\section{References}

[1] Roodman G, Schwartz L. Optimal and heuristic facility phase-out strategies. AIIE Transactions 1975;7:177-84.

[2] Roodman G, Schwartz L. Extensions of the multi-period facility phase-out model: New procedures and applications to a phase-in/phase-out problem. AIIE Transactions 1977;9:103-7.

[3] Van Roy T, Erlenkotter D. A dual-based procedure for dynamic facility location. Management Science 1982;28:1091-105.

[4] Erlenkotter D Pre-investment planning for capacity expansion: a multi-location dynamic model. Ph.D. dissertation. Stanford University, CA, 1967.

[5] Erlenkotter D. Dynamic programming solutions. In: Manne A, editor. Investments for capacity expansion: size, location and time-phasing. Cambridge, MA: MIT Press, 1967. p. 210-27.

[6] Fong C, Srinivasan V. The multi-region dynamic capacity expansion problem: Part II. Operations Research 1981;29:800-16.

[7] Jacobsen S. Multiperiod capacitated location models. In: Mirchandani P, Francis R, editors. Discrete Location Theory. New York: Wiley, 1990. p. 173-208.

[8] Shulman A. An algorithm for solving dynamic capacitated plant location problems with discrete expansion sizes. Operations Research 1991;39:423-36.

[9] Beguin H, Deconnink J, Peeters D. Optimiser la localisation des écoles primaires: Le cas de Mouscron, Belgique. Revue d'Économie Régionale et Urbaine 1989;5:795-806.

[10] Greenleaf N, Harrison T. A mathematical programming approach to elementary school facility decisions. Socio-Economic Planning Sciences 1987;21:395-401.

[11] Henig M, Gershak Y. Dynamic capacity planning of public schools in changing urban communities. SocioEconomic Planning Sciences 1986;20:319-24. 
[12] Pizzolato N. A heuristic for large-size p-median location problems with application to school location. Annals of Operations Research 1994;50:473-85.

[13] Tewari V, Jena S. High school location decision making in rural India and location-allocation models. In: Ghosh A, Rushton G, editors. Spatial analysis and location-allocation models. New York: Van Nostrand Reinhold, 1987. p. 137-62.

[14] Viegas J. Short and mid-term planning of an elementary school network in a suburb of Lisbon. Sistemi Urbani 1987;1:57-77.

[15] Nemhauser G, Wolsey L. Integer and combinatorial optimization. New York: Wiley, 1988.

[16] Winston W. Operations research: applications and algorithms. Belmont, CA: Duxbury Press, 1994.

[17] Antunes A De la planification optimale de L'équipement scolaire (On optimal planning of school networks). Ph.D. dissertation. Université Catholique de Louvain, Belgium, 1994.

[18] Aarts E, Korst J, Laarhoven P. Simulated annealing. In: Aarts E, Leenstra J, editors. Local Search in Combinatorial Optimization. Chichester, UK: Wiley, 1997. p. 91-120.

[19] Cerny V. A thermodynamical approach to the traveling salesman problem. Journal of Optimization Theory and Application 1985;45:41-51.

[20] Kuehn A, Hamburger M. A heuristic program for locating warehouses. Management Science 1963;9:643-66.

[21] Cornuéjols G, Nemhauser G, Wolsey L. The uncapacitated facility location problem. In: Mirchandani P, Francis R, editors. Discrete Location Theory. New York: Wiley, 1990. p. 119-71.

[22] Krarup J, Pruzan P. The simple plant location model: survey and synthesis. European Journal of Operational Research 1983;4:256-69.

[23] Antunes A, Peeters D. On solving a complex location model using simulated annealing algorithm. Submitted for publication.

[24] GEP Educação 1990. Critérios de planeamento da rede escolar (planning criteria for school networks). Ministério da Educação, Lisboa, Portugal.

[25] Hanjoul P, Peeters D. A facility location problem with clients' preferences orderings. Regional Science and Urban Economics 1987;17:451-73. 